\title{
OPTIMAL INTERVALS OF STABILITY OF A FORCED OSCILLATOR
}

\author{
JOSE MIGUEL ALONSO
}

(Communicated by Hal L. Smith)

\begin{abstract}
Consider the differential equation of a nonlinear oscillator with linear friction and a $T$-periodic external force. We find optimal bounds on the derivative of the restoring force and on the period $T$ in order to obtain a unique $T$-periodic solution that is asymptotically stable.
\end{abstract}

\section{INTRODUCTION}

The purpose of this paper is to complete the results obtained in [7] and [1]. Consider the differential equation

$$
x^{\prime \prime}+c x^{\prime}+g(x)=p(t)
$$

where $c>0$ is a fixed constant, $p \in C(\mathbb{R} / T \mathbb{Z})$ and $g \in C^{1}(\mathbb{R})$ satisfies

$$
a \leq g^{\prime}(x) \leq b \text { for each } x \in \mathbb{R}
$$

with $a \geq 0$. If $a=0$ we also need the additional assumption

$$
g(-\infty)<\frac{1}{T} \int_{0}^{T} p(t) d t<g(+\infty) .
$$

Recently, in [7], Ortega has studied the case $a=0$ and obtained sharp conditions on $b$ for the existence, uniqueness and stability of a $T$-periodic solution of (1.1). In fact he has proved that there exists $b_{0}$, that can be computed, such that if $b \leq b_{0}$, then (1.1) has a unique $T$-periodic solution that is (locally) asymptotically stable when (1.2) holds. Moreover there exists $\tau(b)>0$ such that if $b>b_{0}$ but $T \leq \tau(b)$ the above assertion is still true, while if $T>\tau(b)$, then instability will appear for some $p$ satisfying (1.2).

The case $a>0$ was considered in [3] and [4] and sufficient conditions on $a$ and $b$ were obtained for the same problem. More recently, in [1], Ortega and the author have also studied this case and found sharp conditions that guarantee global asymptotic stability (g.a.s.) (and independent from the period $T$ ). In fact we have defined two functions, $A$ and $B$, and we have proved that if $A[a] B[b]<1$, then there exists a unique $T$-periodic solution of $(1.1)$ that is g.a.s., while if $A[a] B[b]>1$, then one can find a periodic function $p$ (for suitable period) such that (1.1) has an unstable periodic solution.

Received by the editors July 1, 1993.

1991 Mathematics Subject Classification. Primary 34C25, 34D20.

(C)1995 American Mathematical Society 
In this paper we shall obtain sharp conditions for a fixed period $T$ when $A[a] B[b] \geq 1$. In fact we shall find a sequence of intervals that can be computed such that if $T$ is in one of these intervals, then (1.1) has a unique $T$-periodic solution that is asymptotically stable. In general this sequence is finite and has several disjoint intervals (see Figure 1). This is different from the case $a=0$ where only one interval appears.

Thanks to the principle of linearized stability the nonlinear problem can be reduced to the following problem in linear theory: to determine under what conditions on $a$ and $b$ the linear equation

$$
y^{\prime \prime}+c y^{\prime}+\alpha(t) y=0 \quad(a \leq \alpha(t) \leq b, \text { a.e. } t \in \mathbb{R})
$$

does not have nontrivial $2 T$-periodic solutions. To deal with this question we shall use a technique based in control theory as used by Brockett in [2] and by Ortega in [7].

The main results on (1.1) are stated in Section 2. The linear equation is studied in Sections 3 and 4. The proofs of the main results appear in Section 5 .

I would like to thank Professor Ortega for encouraging me to study this problem and for his suggestion to elaborate this paper.

\section{THE MAIN THEOREMS}

Consider the equation

$$
x^{\prime \prime}+c x^{\prime}+g(x)=p(t)
$$

where $c>0$ is a fixed constant, $g \in C^{1}(\mathbb{R})$ and $p \in C(\mathbb{R} / T \mathbb{Z})$. It will be assumed that there exist positive constants $a$ and $b$ such that

$$
a \leq g^{\prime}(x) \leq b \quad \forall x \in \mathbb{R} \text {. }
$$

Consider the functions

$$
A[k]= \begin{cases}k^{-1 / 2} \exp \left\{-c \omega_{k}^{-1} \tanh ^{-1}\left(\omega_{k} / c\right)\right\} & \text { if } 0<k<c^{2} / 4 \\ 2 c^{-1} / e & \text { if } k=c^{2} / 4 \\ k^{-1 / 2} \exp \left\{-c \omega_{k}^{-1} \arctan \left(\omega_{k} / c\right)\right\} & \text { if } k>c^{2} / 4\end{cases}
$$

and

$$
B[k]= \begin{cases}0 & \text { if } 0<k \leq c^{2} / 4, \\ k^{1 / 2} \exp \left\{c \omega_{k}^{-1}\left[\arctan \left(\omega_{k} / c\right)-\pi\right]\right\} & \text { if } k>c^{2} / 4,\end{cases}
$$

where $\omega_{k}=\sqrt{\left|4 k-c^{2}\right|}$. Here $\arctan :(-\infty,+\infty) \rightarrow(-\pi / 2, \pi / 2)$. As a consequence of Theorem 1.2 in [1] we have that if $A[a] B[b]<1$, then $(2.1)$ has a unique $T$-periodic solution that is globally asymptotically stable. Therefore we are interested in what happens when $A[a] B[b] \geq 1$.

Theorem 2.1. Suppose that $A[a] B[b] \geq 1$ and (2.2) holds. There exist $\tau_{1}=$ $\tau_{1}(a, b)$ and $\tau_{2}=\tau_{2}(a, b)$ such that if

$$
T \notin n\left[\tau_{1}, \tau_{2}\right] \quad \forall n \in \mathbb{N}
$$

holds, then (2.1) has a unique T-periodic solution that is locally asymptotically stable.

Remark. We shall see below that the constants $\tau_{1}$ and $\tau_{2}$ can be computed. In particular if $A[a] B[b]=1$, then $\tau_{1}=\tau_{2}$ and therefore the intervals of stability 


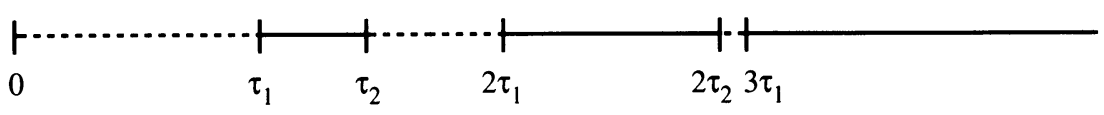

FIGURE 1. The dotted line stands for the intervals of stability in the case $c=2, a=0.3, b=15$. Here $\tau_{1}=1.61408 \ldots$ and $\tau_{2}=2.34997 \ldots$

are the infinite components of $\left\{t>0 \mid t \neq n \tau_{1}, n \in \mathbb{N}\right\}$. On the other hand, if $A[a] B[b]>1$ we have that $\tau_{1}<\tau_{2}$ and hence there must exist some $n \in \mathbb{N}$ such that

$$
\frac{n+1}{n} \leq \frac{\tau_{2}}{\tau_{1}} .
$$

If $n_{0} \in \mathbb{N}$ is the first number satisfying (2.4), then there exist exactly $n_{0}$ intervals of stability for the equation (2.1) (see Figure 1).

In the next result we shall show that (2.3) is sharp. We shall need the additional assumptions

$$
\inf \left\{g^{\prime}(x): x \in \mathbb{R}\right\}=a, \quad \sup \left\{g^{\prime}(x): x \in \mathbb{R}\right\}=b .
$$

Theorem 2.2. Suppose that (2.5) holds. If there exists $n \in \mathbb{N}$ such that

$$
T \in n\left(\tau_{1}, \tau_{2}\right),
$$

then there exists some $p \in C(\mathbb{R} / T \mathbb{Z})$ such that (2.1) has an unstable $T$-periodic solution.

Now we shall say how one can calculate the constants, but first we introduce some notation. If $k>0$ define

$$
\xi_{k}= \begin{cases}\frac{2 \pi}{\omega_{k}} & \text { if } k>c^{2} / 4, \\ +\infty & \text { if } k \leq c^{2} / 4,\end{cases}
$$

where $\omega_{k}=\sqrt{\left|4 k-c^{2}\right|}$. Notice that $\xi_{k}$ is the first positive zero of any nontrivial function $\phi$ satisfying $\phi^{\prime \prime}+c \phi^{\prime}+k \phi=0, \phi(0)=0$. Suppose that $b>c^{2} / 4$ and define the switching function $: \gamma \in \mathrm{L}^{\infty}(\mathbb{R})$ as follows:

$$
\gamma(t)= \begin{cases}a & \text { if } t \leq-\xi_{b}, \\ b & \text { if }-\xi_{b}<t<0, \\ a & \text { if } 0 \leq t\end{cases}
$$

For each $s \in\left[0, \xi_{b}+\xi_{a}\right)$ consider the initial value problem

$$
\left(P_{s}\right)\left\{\begin{array}{l}
y^{\prime \prime}(t)+c y^{\prime}(t)+\gamma(t-s) y(t)=0, \\
y(0)=0, y^{\prime}(0)=1 .
\end{array}\right.
$$

When the solution $y_{s}(t)$ of $\left(P_{s}\right)$ vanishes for some $t>0$, we define $T(s)$ as the first positive zero of $y_{s}(t)$ and define $R(s)=y_{s}^{\prime}(T(s))$ where $y_{s}^{\prime}(t)$ is the derivative of $y_{s}(t)$ with respect to $t$. In the equation the function $\alpha(t)=\gamma(t-s)$ is a piece-wise constant function that at most has one switch in the interval $(0, T(s))$. This equation is similar to the Meissner equation studied for example in $[6$, p. 115] with $c=0$.

The problem $\left(P_{s}\right)$ can be integrated and one can obtain explicitly the functions $T$ and $R$. In particular these functions are defined in an interval $I C$ 


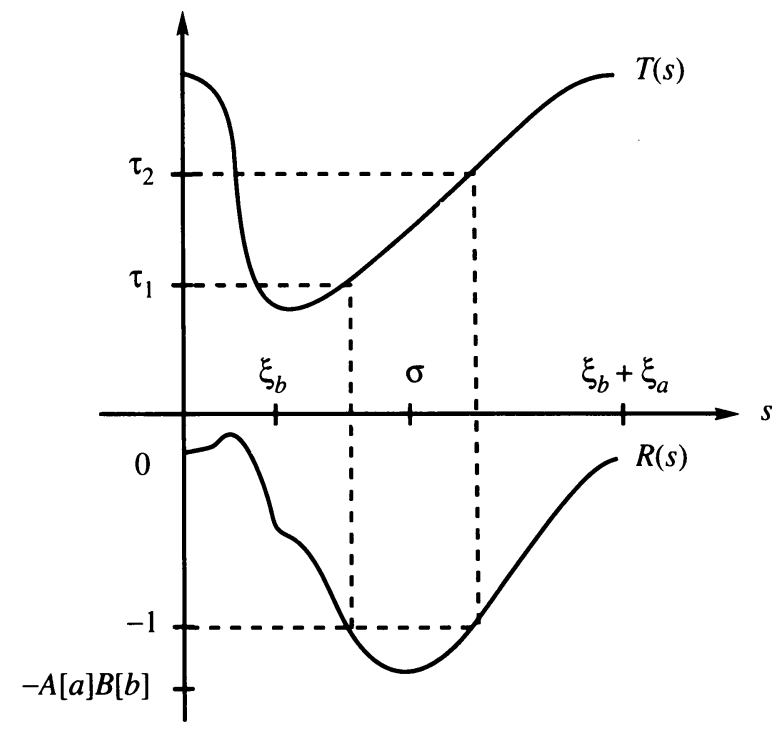

Figure 2. The functions $T$ and $R$ when $a>c^{2} / 4$

$\left[0, \xi_{b}+\xi_{a}\right)$ such that $\left(\xi_{b}, \xi_{b}+\xi_{a}\right) \subset I$. Figure 2 shows the graph of these functions.

The explicit expression of $T(s)$ and $R(s)$ and its properties are given in an appendix at the end of the paper. Then one can compute $\tau_{1}>0$ solving

$$
\tau_{1}=T\left(\bar{s}_{1}\right), \quad R\left(\bar{s}_{1}\right)=-1, \quad \bar{s}_{1} \in\left(\xi_{b}, \sigma\right],
$$

and $\tau_{2}>0$ solving

$$
\tau_{2}=T\left(\bar{s}_{2}\right), \quad R\left(\bar{s}_{2}\right)=-1, \quad \bar{s}_{2} \in\left[\sigma, \xi_{b}+\xi_{a}\right),
$$

where $\sigma$ is the point where $R$ has its minimum. The solvability and uniqueness of these equations follows from Proposition 1 in the appendix.

\section{A PROBLEM IN CONTROL THEORY}

Let $0<a \leq b$ be such that $b>c^{2} / 4$. For $\lambda>0$ consider the problem

$$
\left(P_{\lambda}\right)\left\{\begin{array}{l}
y^{\prime \prime}+c y^{\prime}+\alpha(t) y=0, \\
y(0)=y(L)=0, \quad y^{\prime}(0)=1, \quad y^{\prime}(L)=-\lambda, \\
y(t) \neq 0 \quad \forall t \in(0, L),
\end{array}\right.
$$

where $L>0$ and $\alpha \in \mathrm{L}^{\infty}(0, L)$ is such that

$$
a \leq \alpha(t) \leq b \text { a.e. } t \in(0, L) .
$$

Consider the functions $T(s)$ and $R(s)$ defined on the interval $I$ as in Section 2. We have the following result:

Lemma 3.1. If the problem $\left(P_{\lambda}\right)$ has a solution, then

(i) The equation

$$
R(s)=-\lambda, \quad s \in I,
$$

is solvable. 
(ii) If $s_{1}, s_{2}$ are the solutions of (3.2) and $T\left(s_{1}\right) \leq T\left(s_{2}\right)$, then $L \in$ $\left[T\left(s_{1}\right), T\left(s_{2}\right)\right]$.

Proof. We shall use the language and methods of control theory (see for example [5]). Consider the control process

$$
x^{\prime}=C[u] x, \quad x=\operatorname{col}\left(x_{1}, x_{2}\right), C[u]=\left(\begin{array}{cc}
0 & 1 \\
-u & -c
\end{array}\right) .
$$

The class of admissible controllers is $U=\left\{u \in \mathrm{L}^{\infty}(0, r) \mid r>0, a \leq u(t) \leq\right.$ $b$ a.e. $t \in(0, r)\}$, the initial point is $X_{0}=\operatorname{col}(0,1)$ and the target set is $K=\{(0, d) \mid d \leq 0\}$. For each $u \in U$ attaining the target set denote by $\tau(u)$ the first positive zero of $x_{1}(t)$, where $x=\operatorname{col}\left(x_{1}, x_{2}\right)$ is the corresponding response. Consider the set $V=\left\{u \in U \mid x_{2}(\tau(u))=-\lambda\right\}$. Note that the lemma will be proved if there exist $s_{1}, s_{2} \in I$ such that $T(V) \subset\left[T\left(s_{1}\right), T\left(s_{2}\right)\right]$ and $R\left(s_{1}\right)=R\left(s_{2}\right)=-\lambda$.

To prove this consider for each $n \in \mathbb{N}$ the cost functional

$$
F_{n}[u]=\tau(u)+n\left(x_{2}(\tau(u))+\lambda\right)^{2} .
$$

Note that $\alpha \in V$ and that if $v \in V$, then $F_{n}[v]=\tau(v)$. Let $u_{n}^{*}$ be an optimal control minimizing $F_{n}$ with optimal response $x_{n}^{*}=\operatorname{col}\left(x_{1}^{* n}, x_{2}^{* n}\right)$. Its existence follows for example from Theorem 4 in [5, p. 259]. Consider the Hamiltonian function $H(\eta, x, u)=\eta \cdot C[u] x=\left(\eta_{1}-c \eta_{2}\right) x_{2}-u \eta_{2} x_{1}$, and the function $M(\eta, x)=\max \{H(\eta, x, u) \mid a \leq u \leq b\}=\left(\eta_{1}-c \eta_{2}\right) x_{2}+b\left(\eta_{2} x_{1}\right)^{-}-a\left(\eta_{2} x_{1}\right)^{+}$. The maximal principle of Pontryagin says that there exists $\bar{\eta}=\operatorname{col}\left(\bar{\eta}_{1}, \bar{\eta}_{2}\right)$ a nontrivial solution of $\eta^{\prime}=-C\left[u_{n}^{*}\right]^{\tau} \eta$ such that $H\left(\bar{\eta}, x_{n}^{*}, u_{n}^{*}\right)=M\left(\bar{\eta}, x_{n}^{*}\right)$ a.e. $t \in\left(0, \tau\left(u_{n}^{*}\right)\right)$. In consequence

$$
u_{n}^{*}(t)= \begin{cases}a & \text { if } \bar{\eta}_{2}(t)>0 \\ b & \text { if } \bar{\eta}_{2}(t)<0\end{cases}
$$

Since $\bar{\eta}_{2}(t)$ and $x_{1}^{* n}(t)$ are solutions of adjoint equations and $x_{1}^{* n}(t) \neq 0 \forall t \in$ $\left(0, \tau\left(u_{n}^{*}\right)\right)$ it follows that $\bar{\eta}_{2}(t)$ has at most one zero in $\left(0, \tau\left(u_{n}^{*}\right)\right)$. Therefore $u_{n}^{*}$ has at most one jump in $\left(0, \tau\left(u_{n}^{*}\right)\right)$ and only takes the values $a$ and $b$. Hence $u_{n}^{*}$ is a switching function as considered in Section 2 and there must exist $s_{n}^{*}$ such that $u_{n}^{*}(t)=\gamma\left(t-s_{n}^{*}\right)$ a.e. $t \in\left(0, \tau\left(u_{n}^{*}\right)\right)$. Moreover $\tau\left(u_{n}^{*}\right)=T\left(s_{n}^{*}\right)$ and $x_{2}^{* n}\left(\tau\left(u_{n}^{*}\right)\right)=R\left(s_{n}^{*}\right)$.

The sequence $\left\{s_{n}^{*}\right\}$ is bounded. This follows from

$$
T\left(s_{n}^{*}\right)=\tau\left(u_{n}^{*}\right) \leq F_{n}\left[u_{n}^{*}\right] \leq F_{n}[\alpha]=\tau(\alpha)=L
$$

and the properties of $T(s)$ (see Proposition 1 (ii) in the appendix). Hence we can suppose that $\left\{s_{n}^{*}\right\}$ converge (in the other case one can take a convergent subsequence). If $a \leq c^{2} / 4$, then (3.3) implies that the limit of $\left\{s_{n}^{*}\right\}$ is in $I$. If $a>c^{2} / 4$, then $I=\left[0, \xi_{b}+\xi_{a}\right)$ but $T(0)=T\left(\xi_{b}+\xi_{a}\right)$ and $R(0)=R\left(\xi_{b}+\xi_{a}\right)$. Therefore in both cases there exists $s_{1} \in I$ such that $T\left(s_{n}^{*}\right) \rightarrow T\left(s_{1}\right)$ and $R\left(s_{n}^{*}\right) \rightarrow R\left(s_{1}\right)$. Let $x^{*}=\operatorname{col}\left(x_{1}^{*}, x_{2}^{*}\right)$ be the solution of $x^{\prime}=C\left[\gamma\left(t-s_{1}\right)\right] x$, $x(0)=\operatorname{col}(0,1)$. Note that $T\left(s_{1}\right)=\tau\left(\gamma\left(\cdot-s_{1}\right)\right)$ and $R\left(s_{1}\right)=x_{2}^{*}\left(T\left(s_{1}\right)\right)$.

We assert that $R\left(s_{1}\right)=-\hat{\lambda}$. If the claim is not true there must exist a subsequence such that $\left(R\left(s_{n_{k}}^{*}\right)+\lambda\right) \rightarrow r \neq 0$. But then $F_{n_{k}}\left[u_{n_{k}}^{*}\right]=T\left(s_{n_{k}}^{*}\right)+$ $n_{k}\left(R\left(s_{n_{k}}^{*}\right)+\lambda\right)^{2} \rightarrow+\infty$ contradicting that $u_{n_{k}}^{*}$ is the minimal optimal control for $F_{n_{k}}$ since $F_{n_{k}}[\alpha]=L<+\infty$. 
Finally, if $v \in V$, then $\tau(v) \geq T\left(s_{1}\right)$. In the other case, since $T\left(s_{n}^{*}\right) \rightarrow$ $T\left(s_{1}\right)$, for $n$ sufficiently large we should have $F_{n}[v]=\tau(v) \leq T\left(s_{n}^{*}\right) \leq F_{n}\left[u_{n}^{*}\right]$ contradicting again the optimality of $u_{n}^{*}$.

To calculate $s_{2}$ we can use the same reasoning minimizing the cost functional

$$
G_{n}[u]=e^{-\tau(u)}+n\left(x_{2}(\tau(u))+\lambda\right)^{2}
$$

for $n \in \mathbb{N}$ such that $1+n \lambda^{2}>e^{-L}$.

For the next result suppose that $A[a] B[b] \geq 1$. Then as consequence of Proposition 1 in the appendix there exist unique $\tau_{1}>0$ and $\tau_{2}>0$ satisfying (2.7) and (2.8).

Lemma 3.2. Suppose $A[a] B[b] \geq 1$ and let $\tau_{1}, \tau_{2}$ be as in (2.7) and (2.8).

(i) If the problem $\left(P_{1}\right)$ has a solution, then $L \in\left[\tau_{1}, \tau_{2}\right]$.

(ii) If $\widetilde{L} \in\left[\tau_{1}, \tau_{2}\right]$, then there exists $\tilde{\alpha} \in L^{\infty}(0, \widetilde{L})$ satisfying (3.1) such that the corresponding problem $\left(\widetilde{P}_{1}\right)$ has a solution.

Proof. (i) This is a particular case of Lemma 3.1.

(ii) If $\tilde{L} \in\left[\tau_{1}, \tau_{2}\right]$ there exists $\tilde{s} \in\left[\bar{s}_{1}, \bar{s}_{2}\right]$ such that $T(\tilde{s})=\tilde{L}$. Then consider $\tilde{\alpha}(t)=\gamma(t-\tilde{s})$ and the problem

$$
\left(\widetilde{P}_{1}\right)\left\{\begin{array}{l}
y^{\prime \prime}+c y^{\prime}+\tilde{\alpha}(t) y=0, \\
y(0)=y(\widetilde{L})=0, \quad y^{\prime}(0)=1, \quad y^{\prime}(\widetilde{L})=-1, \\
y(t) \neq 0 \text { in }(0, \widetilde{L})
\end{array}\right.
$$

has a solution.

Corollary 3.3. With the same conditions as in the previous lemma, if $T \in$ $\left(\tau_{1}, \tau_{2}\right)$, then there exists $\beta \in \mathrm{L}^{\infty}(\mathbb{R} / T \mathbb{Z})$ satisfying (3.1) such that the equation

$$
y^{\prime \prime}+c y^{\prime}+\beta(t) y=0
$$

is unstable.

Proof. If $T \in\left(\tau_{1}, \tau_{2}\right)$, then $A[a] B[b]>1$ and there exists $s \in\left(\bar{s}_{1}, \bar{s}_{2}\right)$ such that $T(s)=T$. Consider $\beta \in \mathrm{L}^{\infty}(\mathbb{R} / T \mathbb{Z})$ such that $\beta(t)=\gamma(t-s) \forall t \in(0, T)$. The solution $y(t)$ of (3.4) satisfies $y(t+T)=R(s) y(t) \forall t \in \mathbb{R}$. Then $R(s)<-1$ is a Floquet multiplier of (3.4) and thus is unstable.

\section{THE LINEAR EQUATION}

Let $0<a \leq b$ be such that $A[a] B[b] \geq 1$. Consider the linear equation

$$
y^{\prime \prime}+c y^{\prime}+\alpha(t) y=0
$$

where $\alpha \in \mathrm{L}^{\infty}(\mathbb{R} / T \mathbb{Z})$ satisfies

$$
a \leq \alpha(t) \leq b \text { a.e. } t \in \mathbb{R} .
$$

Proposition 4.1. Let $\tau_{1} \leq \tau_{2}$ be as in (2.7) and (2.8) respectively. If

$$
T \notin n\left[\tau_{1}, \tau_{2}\right] \quad \forall n \in \mathbb{N},
$$

then there does not exist any nontrivial 2T-periodic solution of (4.1).

Proof. Suppose, contrary to the assertion of the proposition, that there exists a nontrivial $2 T$-periodic solution of (4.1). If $\phi(t)$ is such a solution, then it must 
vanish (to see this integrate (4.1) over a period). Let $t_{0}$ be such that $\phi\left(t_{0}\right)=0$. Since the zeros of $\phi$ are simple and $\phi$ is periodic, the number of zeros in the interval $\left[t_{0}, t_{0}+2 T\right)$ is even. Let $2 n(n \in \mathbb{N})$ be the number of zeros of $\phi$ in $\left[t_{0}, t_{0}+2 T\right)$.

We shall again use the language of control theory, but now we use polar coordinates: $y_{1}(t)=\rho(t) \sin \theta(t), y_{2}(t)=\rho(t) \cos \theta(t)$. Consider the control process

$$
\left\{\begin{array}{l}
\theta^{\prime}=\cos ^{2} \theta+u \sin ^{2} \theta+c \sin \theta \cos \theta, \\
\rho^{\prime}=\rho\left((1-u) \sin \theta \cos \theta-c \cos ^{2} \theta\right) .
\end{array}\right.
$$

The class of admissible controllers is $U=\left\{u \in \mathrm{L}^{\infty}\left(t_{0}, r\right) \mid r>t_{0}, a \leq u(t) \leq b\right.$ a.e. $\left.t \in\left(t_{0}, r\right)\right\}$, the initial point is $X_{0}=\operatorname{col}(0,1)$ and the target state is $X_{1}=\operatorname{col}(2 n \pi, 1)$. Consider the control problems of minimal and maximal time to attain the target state. Note that if $\operatorname{col}(\theta(t), \rho(t))$ is a solution of (4.4), then $y(t)=\rho(t) \sin \theta(t)$ is a solution of (4.1). Also note that $\theta(t)$ is increasing when $\theta(t)=j \pi, j \in \mathbb{Z}$. Let $u^{*}$ and $\operatorname{col}\left(\rho^{*}, \lambda^{*}\right)$ be a minimal optimal control and the corresponding response. Let

$$
t_{0}<t_{1}<\cdots<t_{2 n}=\tau^{*}
$$

be such that $\theta^{*}\left(t_{k}\right)=k \pi \quad(k=1, \ldots, 2 n)$. Observe that $\rho^{*}\left(t_{0}\right)=\rho^{*}\left(t_{2 n}\right)=1$. As a consequence of the principle of optimability and Lemma 3.1 there exist $s_{1}^{*}<\cdots<s_{2 n}^{*}$ such that $R\left(s_{k}^{*}\right)=-\rho^{*}\left(t_{k}\right) / \rho^{*}\left(t_{k-1}\right)$ and $t_{k}-t_{k-1}=T\left(s_{k}^{*}\right)$ for each $k=1, \ldots, 2 n$. Hence we have $\tau^{*}=T\left(s_{1}^{*}\right)+\cdots+T\left(s_{2 n}^{*}\right)$ and

$$
R\left(s_{1}^{*}\right) \cdots R\left(s_{2 n}^{*}\right)=1
$$

Thus $\left(s_{1}^{*}, \ldots, s_{2 n}^{*}\right)$ is a solution of the problem of minimizing the function $f\left(s_{1}, \ldots, s_{2 n}\right)=T\left(s_{1}\right)+\cdots+T\left(s_{2 n}\right)$ in the set $C=\left\{\left(s_{1}, \ldots, s_{2 n}\right) \mid g\left(s_{1}, \ldots, s_{2 n}\right)\right.$ $\left.=0, s_{k} \in I \forall k\right\}$ where $g\left(s_{1}, \ldots, s_{2 n}\right)=R\left(s_{1}\right) \cdots R\left(s_{2 n}\right)-1$. Let $\sigma$ be as in Section 2. From (4.5) and Proposition 1(iii) in the appendix we have that $R(\sigma) \geq 1$. We distinguish two cases:

Case 1: $|R(\sigma)|=1$. This happens when $A[a] B[b]=1$. Furthermore $\tau_{1}=$ $T(\sigma)$ and $C=\{(\sigma, \ldots, \sigma)\}$. Hence $s_{1}^{*}=\cdots=s_{2 n}^{*}=\sigma$ and $\tau^{*}=2 n \tau_{1}$.

Case 2: $|R(\sigma)|>1$. Lemma 2 and Proposition 1 (iv) in the appendix imply $s_{k}^{*} \in\left(\xi_{b}, \xi_{b}+\xi_{a}\right)$. Now by using Lagrange's multipliers there exists $\lambda \in \mathbb{R}$ such that $\nabla f\left(s_{1}^{*}, \ldots, s_{2 n}^{*}\right)=\lambda \nabla g\left(s_{1}^{*}, \ldots, s_{2 n}^{*}\right)$, or equivalently

$$
\begin{aligned}
T^{\prime}\left(s_{1}^{*}\right) & =\lambda R^{\prime}\left(s_{1}^{*}\right) \cdots R\left(s_{2 n}^{*}\right) \\
& \cdots \cdots \cdots \cdots \\
T^{\prime}\left(s_{2 n}^{*}\right) & =\lambda R\left(s_{1}^{*}\right) \cdots R^{\prime}\left(s_{2 n}^{*}\right) .
\end{aligned}
$$

As in this case $T^{\prime}(\sigma) \neq 0$ and $R^{\prime}(\sigma)=0$ one has that $s_{k}^{*} \neq \sigma$ and hence $R^{\prime}\left(s_{k}^{*}\right) \neq 0$ for each $k=1, \ldots, 2 n$. Thus one car divide each equation by the corresponding $R^{\prime}\left(s_{k}^{*}\right)$ and multiply by $R\left(s_{k}^{*}\right)$ to obtain

$$
\frac{T^{\prime}\left(s_{1}^{*}\right) R\left(s_{1}^{*}\right)}{R^{\prime}\left(s_{1}^{*}\right)}=\cdots=\frac{T^{\prime}\left(s_{2 n}^{*}\right) R\left(s_{2 n}^{*}\right)}{R^{\prime}\left(s_{2 n}^{*}\right)}=\lambda
$$

thanks to (4.5). Now, since the function $h(s)=T^{\prime}(s) R(s) / R^{\prime}(s)$ is one-to-one in $\left(\xi_{b}, \xi_{b}+\xi_{a}\right) \backslash\{\sigma\}$, then $s_{1}^{*}=\cdots=s_{2 n}^{*}$. Hence $R\left(s_{1}^{*}\right)^{2 n}=1$ and consequently we have that $s_{1}^{*}=\bar{s}_{1}$ and $\tau^{*}=2 n \tau_{1}$. 
Therefore in both cases the minimal optimal time is $2 n \tau_{1}$. We use the same reasoning to show that the maximal optimal time is $2 n \tau_{2}$. But since $u=\alpha$ is a control that allows one to reach the target at the time $2 T$ we have $2 n \tau_{1} \leq 2 T \leq 2 n \tau_{2}$ leading to $n \tau_{1} \leq T \leq n \tau_{2}$, contradicting (4.3).

\section{Proofs of THE MAIN THEOREMS}

Now the results in Section 2 follow from standard methods:

Proof of Theorem 2.1. Repeating the reasoning of [8, p. 168] one obtains that (2.1) has at least one $T$-periodic solution. If $x_{1}$ and $x_{2}$ are $T$-periodic solutions of $(2.1)$, then $y(t)=x_{1}(t)-x_{2}(t)$ is a $T$-periodic solution of (4.1) with

$$
\alpha(t)= \begin{cases}\frac{g\left(x_{1}(t)\right)-g\left(x_{2}(t)\right)}{x_{1}(t)-x_{2}(t)} & \text { if } x_{1}(t) \neq x_{2}(t), \\ a & \text { if } x_{1}(t)=x_{2}(t),\end{cases}
$$

that satisfies (4.2). From Proposition 4.1 one obtains that $y \equiv 0$ and hence (2.1) has a unique $T$-periodic solution. If $z(t)$ is such a solution, consider for each $s \in[0,1]$ the linear equation

$$
y^{\prime \prime}(t)+c y^{\prime}(t)+\left[s g^{\prime}(z(t))+a(1-s)\right] y(t)=0 .
$$

Note that for $s=1(5.1)$ is the linearized equation of (2.1) at $z(t)$. Let $\Delta[s]$ be the discriminant of $(5.1)$, that is, the trace of a monodromy matrix. This is a continuous function of $s$ (see for instance Lemma 2.1 in [7]) and it is well known that the existence of $2 T$-periodic solutions of $(5.1)$ is equivalent to

$$
|\Delta[s]|=1+e^{-c T} .
$$

Therefore from Proposition 4.1 it follows that $|\Delta[s]| \neq 1+e^{-c T}$ and since $|\Delta[0]|<1+e^{-c T}$ one obtains that $|\Delta[s]|<1+e^{-c T}$ for each $s \in[0,1]$. Therefore the Floquet multipliers lie on the open unit disk. In particular this is true for $s=1$ and the asymptotic stability of $z(t)$ is a consequence of the principle of linearized stability.

Proof of Theorem 2.2. Since $T / n \in\left(\tau_{1}, \tau_{2}\right)$ one can use the same argument of [7, Theorem II] together with Corollary 3.3 to state the theorem.

\section{APPENDIX}

Here we study the properties of the functions $T(s)$ and $R(s)$ defined in Section 2 related to the linear initial value problem $\left(P_{s}\right)$.

Proposition 1. (i) $T(s)$ and $R(s)$ are $C^{1}$ functions defined in an interval $I \subset$ $\left[0, \xi_{b}+\xi_{a}\right)$ such that $\left(\xi_{b}, \xi_{b}+\xi_{a}\right) \subset I$.

(ii) $T(s)$ is strictly increasing in $\left(\xi_{b}, \xi_{b}+\xi_{a}\right)$. Moreover if $a \leq c^{2} / 4$, then

$$
\lim _{s \rightarrow \text { inf } I} T(s)=\lim _{s \rightarrow \text { sup } I} T(s)=+\infty .
$$

(iii) $R^{\prime}(s)$ has only one zero in $\left(\xi_{b}, \xi_{b}+\xi_{a}\right)$ where $R(s)$ reaches a minimum. If $\sigma$ is such a zero, then $R(\sigma)=-A[a] B[b]$.

(iv) If $s \notin\left(\xi_{b}, \xi_{b}+\xi_{a}\right)$, then $|R(s)|<1$.

(v) The equation

$$
R(s)=r, \quad s \in I,
$$

has at most two solutions for each $r \in \mathbb{R}$. 
Proof. After a simple but tedious computation one can obtain explicitly the functions $T$ and $R$, the interval $I$ and the minimum $\sigma$. We distinguish three cases. In all cases the properties follow from simple verification.

Case 1: $a>c^{2} / 4$. Then $I=\left[0, \xi_{b}+\xi_{a}\right)$ and

$$
\begin{aligned}
& T(s)= \begin{cases}s+\frac{2}{\omega_{a}}\left(\pi-\arctan \left(\frac{\omega_{a}}{\omega_{b}} \tan \left(\frac{\omega_{b}}{2} s\right)\right)\right) & \text { if } 0 \leq s<\frac{\pi}{\omega_{b}}, \\
\frac{\pi}{\omega_{a}}+\frac{\pi}{\omega_{b}} & \text { if } s=\frac{\pi}{\omega_{b}}, \\
s-\frac{2}{\omega_{a}} \arctan \left(\frac{\omega_{a}}{\omega_{b}} \tan \left(\frac{\omega_{b}}{2} s\right)\right) & \text { if } \frac{\pi}{\omega_{b}}<s \leq \xi_{b}, \\
s-\xi_{b}+\frac{2}{\omega_{b}}\left(\pi-\arctan \left(\frac{\omega_{b}}{\omega_{a}} \tan \left(\frac{\omega_{a}}{2}\left(s-\xi_{b}\right)\right)\right)\right) & \text { if } \xi_{b}<s<\xi_{b}+\frac{\pi}{\omega_{a}}, \\
\frac{\pi}{\omega_{a}}+\frac{\pi}{\omega_{b}} & \text { if } s=\xi_{b}+\frac{\pi}{\omega_{b}}, \\
s-\xi_{b}-\frac{2}{\omega_{b}} \arctan \left(\frac{\omega_{b}}{\omega_{a}} \tan \left(\frac{\omega_{a}}{2}\left(s-\xi_{b}\right)\right)\right) & \text { if } \xi_{b}+\frac{\pi}{\omega_{a}}<s<\xi_{b}+\xi_{a},\end{cases} \\
& R(s)= \begin{cases}-\sqrt{\cos ^{2}\left(\frac{\omega_{b}}{2} s\right)+\left(\frac{\omega_{a}}{\omega_{b}}\right)^{2} \sin ^{2}\left(\frac{\omega_{b}}{2} s\right) e^{-\frac{c}{2} T(s)}} & \text { if } 0 \leq s<\xi_{b}, \\
-\sqrt{\cos ^{2}\left(\frac{\omega_{a}}{2}\left(s-\xi_{b}\right)\right)+\left(\frac{\omega_{b}}{\omega_{a}}\right)^{2} \sin ^{2}\left(\frac{\omega_{a}}{2}\left(s-\xi_{b}\right)\right)} e^{-\frac{c}{2} T(s)} & \text { if } \xi_{b} \leq s<\xi_{b}+\xi_{a} .\end{cases}
\end{aligned}
$$

$R(s)$ has its minimum in $\sigma=\xi_{b}+2 \arctan \left(\omega_{a} / c\right) / \omega_{a}$. Figure 2 is a drawing of these functions in this case.

Case 2: $a=c^{2} / 4$. Then $I=\left(\pi / \omega_{b},+\infty\right)$ and

$$
\begin{gathered}
T(s)= \begin{cases}s-\frac{2}{\omega_{b}} \tan \left(\frac{\omega_{b}}{2} s\right) & \text { if } \frac{\pi}{\omega_{b}}<s \leq \xi_{b}, \\
s-\xi_{b}+\frac{2}{\omega_{b}}\left(\pi-\arctan \left(\frac{\omega_{b}}{2}\left(s-\xi_{b}\right)\right)\right) & \text { if } \xi_{b} \leq s,\end{cases} \\
R(s)= \begin{cases}\cos \left(\frac{\omega_{b}}{2} s\right) e^{-\frac{c}{2} T(s)} & \text { if } \frac{\pi}{\omega_{b}}<s \leq \xi_{b}, \\
-\frac{1}{2} \sqrt{4+\omega_{b}^{2}\left(s-\xi_{b}\right)^{2}} e^{-\frac{c}{2} T(s)} & \text { if } \xi_{b} \leq s .\end{cases}
\end{gathered}
$$

Here $R(s)$ has its minimum in $\sigma=\xi_{b}+2 / c$.

Case 3: $a<c^{2} / 4$. Then $I=\left(2\left(\pi-\arctan \left(\omega_{b} / \omega_{a}\right)\right) / \omega_{b},+\infty\right)$ and

$$
\begin{aligned}
& T(s)=\left\{\begin{array}{l}
s-\frac{2}{\omega_{a}} \tanh ^{-1}\left(\frac{\omega_{a}}{\omega_{b}} \tan \left(\frac{\omega_{b}}{2} s\right)\right) \quad \text { if } \frac{2}{\omega_{b}}\left(\pi-\arctan \left(\frac{\omega_{b}}{\omega_{a}}\right)\right)<s \leq \xi_{b}, \\
s-\xi_{b}+\frac{2}{\omega_{b}}\left(\pi-\arctan \left(\frac{\omega_{b}}{\omega_{a}} \tanh \left(\frac{\omega_{a}}{2}\left(s-\xi_{b}\right)\right) \quad \text { if } \xi_{b} \leq s,\right.\right.
\end{array}\right. \\
& R(s)=\left\{\begin{array}{l}
-\sqrt{\cos ^{2}\left(\frac{\omega_{b}}{2} s\right)-\left(\frac{\omega_{a}}{\omega_{b}}\right)^{2} \sin ^{2}\left(\frac{\omega_{b}}{2} s\right)} e^{-\frac{c}{2} T(s) \quad \text { if } \frac{2}{\omega_{b}}\left(\pi-\arctan \left(\frac{\omega_{b}}{\omega_{a}}\right)\right)<s \leq \xi_{b},} \\
-\sqrt{\cosh ^{2}\left(\frac{\omega_{a}}{2}\left(s-\xi_{b}\right)\right)+\left(\frac{\omega_{b}}{\omega_{a}}\right)^{2} \sinh ^{2}\left(\frac{\omega_{a}}{2}\left(s-\xi_{b}\right)\right)} e^{-\frac{c}{2} T(s) \quad \text { if } \xi_{b} \leq s .}
\end{array}\right.
\end{aligned}
$$

$R(s)$ has its minimum in $\sigma=\xi_{b}+2 \tanh ^{-1}\left(\omega_{a} / c\right) / \omega_{a}$.

In Section 4 we used the following lemma.

Lemma 2. (i) The function $h(s)=R(s) T^{\prime}(s) / R^{\prime}(s)$ is one-to-one on $\left(\xi_{b}, \xi_{b}+\right.$ $\left.\xi_{a}\right) \backslash\{\sigma\}$. Let $s_{1}, s_{2} \in I$ be such that $s_{1} \notin\left(\xi_{b}, \xi_{b}+\xi_{a}\right)$ and $\left|R\left(s_{2}\right)\right|>1$. Then

(ii) There exist $\tilde{s}_{1}, \tilde{s}_{2} \in I$ such that $R\left(\tilde{s}_{1}\right) R\left(\tilde{s}_{2}\right)=R\left(s_{1}\right) R\left(s_{2}\right)$ and $T\left(\tilde{s}_{1}\right)+$ $T\left(\tilde{s}_{2}\right)<T\left(s_{1}\right)+T\left(s_{2}\right)$.

(iii) There exist $\hat{s}_{1}, \hat{s}_{2} \in I$ such that $R\left(\hat{s}_{1}\right) R\left(\hat{s}_{2}\right)=R\left(s_{1}\right) R\left(s_{2}\right)$ and $T\left(\hat{s}_{1}\right)+$ $T\left(\hat{s}_{2}\right)>T\left(s_{1}\right)+T\left(s_{2}\right)$.

Proof. We study the case $a>c^{2} / 4$. The other cases are similar and are left to the reader. 
(i) Here $h(s)=2 \sin \left(\omega_{a}\left(s-\xi_{b}\right) / 2\right) /\left(\omega_{a} \cos \left(\omega_{a}\left(s-\xi_{b}\right) / 2\right)-c \sin \left(\omega_{a}\left(s-\xi_{b}\right) / 2\right)\right)$ is strictly increasing in $\left(\xi_{b}, \xi_{b}+\xi_{a}\right) \backslash\{\sigma\}$.

(ii) Let $\sigma_{0}=2 \arctan \left(\omega_{b} / c\right) / \omega_{b} . R(s)$ reaches a maximum in $\sigma_{0}$. We distinguish several cases. If $s_{1} \in\left[0, \sigma_{0}\right)$, then there exists $\tilde{s}_{1} \in\left(\sigma_{0}, \xi_{b}\right)$ such that $R\left(\tilde{s}_{1}\right)=R\left(s_{1}\right)$ and $T\left(\tilde{s}_{1}\right)<T\left(s_{1}\right)$ (see Figure 2). Similarly, if $s_{2} \in\left(\sigma, \xi_{b}+\right.$ $\left.\xi_{a}\right)$, then there exists $\tilde{s}_{2} \in\left(\xi_{b}, \sigma\right)$ such that $R\left(\tilde{s}_{2}\right)=R\left(s_{2}\right)$ and $T\left(\tilde{s}_{2}\right)<T\left(s_{2}\right)$, proving the assertion.

Therefore suppose that $s_{1} \in\left[\sigma_{0}, \xi_{b}\right]$ and $s_{2} \in\left[\xi_{b}, \sigma\right]$. Since $R(s)$ is strictly decreasing in $\left[\sigma_{0}, \sigma\right]$, for each $s \in\left[s_{1}, s_{2}\right]$ there exists a unique $\varphi(s)$ such that $R(s) R(\varphi(s))=R\left(s_{1}\right) R\left(s_{2}\right)$. The function $\varphi(s)$ is continuous and decreasing. Moreover $T(s)$ is strictly decreasing in $\left[\sigma_{0}, \xi_{b}\right]$ and increasing in $\left[\xi_{b}, \sigma\right]$. In consequence we obtain (ii) for $s_{1} \in\left[\sigma_{0}, \xi_{b}\right)$ taking $\tilde{s}_{1}=s, \tilde{s}_{2}=\varphi(s)$ for any $s \in\left(\sigma_{0}, \xi_{b}\right)$.

Finally suppose that $s_{1}=\xi_{b}$. Consider the function $f(s)=T(s)+T(\varphi(s))$, $s \in\left[s_{1}, s_{2}\right]$, and let $r \in\left(\xi_{b}, s_{2}\right)$ be such that $r=\varphi(r)$. We assert that $f(s)$ is decreasing in $\left(\xi_{b}, r\right)$. Note that this implies (ii). From the inverse function theorem, $\varphi(s)$ is differentiable in $\left(\xi_{b}, r\right)$ and

$$
\varphi^{\prime}(s)=-\frac{R^{\prime}(s) R(\varphi(s))}{R(s) R^{\prime}(\varphi(s))}
$$

also $f(s)$ is differentiable and $f^{\prime}(s)=T^{\prime}(s)+T^{\prime}(\varphi(s)) \varphi^{\prime}(s)$. Multiplying by $R(s) / R^{\prime}(s)>0$ one obtains

$$
\frac{R(s)}{R^{\prime}(s)} f^{\prime}(s)=h(s)-h(\varphi(s))<0
$$

and hence $f^{\prime}(s)<0$ in $\left(\xi_{b}, r\right)$, proving the assertion.

(iii) Similar.

\section{REFERENCES}

1. J. M. Alonso and R. Ortega, Boundedness and global asymptotic stability of a forced oscillator, Nonlinear Anal. TMA (to appear).

2. R. W. Brockett, Variational methods for stability of periodic equations, Differential Equations and Dynamical Systems (J. Hale and J. Lasalle, eds.), Academic Press, New York, 1967, pp. 299-308.

3. A. C. Lazer and P. J. McKenna, Existence, uniqueness, and stability of oscillations in differential equations with asymmetric nonlinearities, Trans. Amer. Math. Soc. 315 (1989), 721-739.

4. Proc. Amer. Math. Soc. 110 (1990), 125-133.

5. E. Lee and L. Markus, Foundations of optimal control theory, Wiley, New York, 1967.

6. W. Magnus and S. Winkler, Hill's equation, Dover, New York, 1979.

7. R. Ortega, The first interval of stability of a periodic equation of Duffing type, Proc. Amer. Math. Soc. 115 (1992), 1061-1067.

8. T. Yoshizawa, Stability theory and the existence of periodic solutions and almost periodic solutions, Springer-Verlag, Berlin, 1975.

Departamento de Matematica Aplicada, facultad de Ciencias, Universidad de Granada, 18071 Granada, Spain

E-mail address: jalonso@ugr.es 